The Last Word

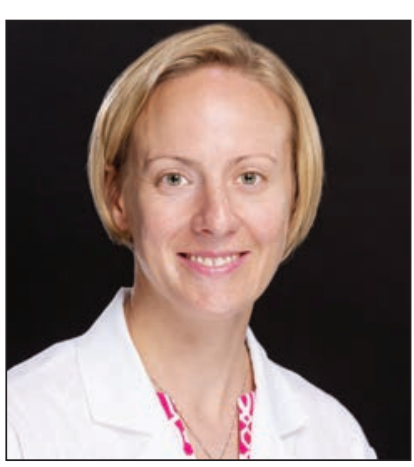

Bethany L. Niell, MD, PhD

Bethany L. Niell, MD, PhD, is an Associate Member at Moffitt Cancer Center. Dr. Niell received her degrees from the University of Michigan. She completed a Diagnostic Radiology Residency at Massachusetts General Hospital, where she also served as Chief Resident. Dr. Niell is a boardcertified, subspecialty trained diagnostic radiologist. Her clinical practice involves interpreting mammograms, ultrasound, and breast MRI and performing image-guided breast biopsies. Dr. Niell is especially interested in the appropriate utilization of breast imaging, including breast MRI, mammography, and tomosynthesis, based on her background in public health.

\section{Appropriate Use of Imaging to Detect Primary, Second Primary, and Recurrent Breast Cancer}

\author{
Bethany L. Niell, MD, PhD
}

Within the United States, approximately 250,000 new female breast cancers will be diagnosed each year, and among women diagnosed between 2008 and 2014, 89.7\% have survived $\geq 5$ years. ${ }^{1}$ For the $62 \%$ of women diagnosed with localized disease, $>98 \%$ will be alive 5 years after diagnosis. ${ }^{1}$ As a result, $>3.4$ million women in the United States are living with a breast cancer diagnosis. ${ }^{1}$ Many of these women may undergo surveillance imaging for recurrent, metastatic, or second primary breast cancer. However, the progression from active treatment to survivorship for these women may be fraught with difficulty because of transitions in the healthcare team, complicating comorbid conditions, and inadequate access to high-quality care due to socioeconomic disparities. ${ }^{2}$ This may result in suboptimal care of survivors, including inappropriate use of imaging examinations.

In "Geographic Variation in Postoperative Imaging for Low-Risk Breast Cancer," published in this issue, Franc et al report geographic variations in breast and nonbreast imaging studies in breast cancer survivors. Using a database with information from approximately 100 insurance companies, the authors reviewed claims data of women with unilateral invasive breast cancer who were diagnosed with a breast biopsy in 2010 through 2012. Claims for high-cost imaging studies, such as CT, brain or body MRI, PET, and bone scans, in the 18-month postoperative period were included if the imaging was performed for a breast cancer indication.

The authors attempted to limit the analysis to early-stage breast cancers by excluding women who received neoadjuvant or adjuvant chemotherapy. Use of at least 1 imaging study and imaging intensity (defined as the mean number of imaging studies per patient per year) were analyzed in the final cohort of 36,045 women. Analyses were stratified by geographic region, as determined by metropolitan statistical area (MSA), insurance class, and treatment regimen (eg, lumpectomy \pm radiation therapy $[R T]$, mastectomy $\pm \mathrm{RT}$ ). In the 18 months after diagnosis, $69 \%$ of women underwent mammography, although the authors included women who received a mastectomy or contralateral mastectomy and at least a subset of these women would not be expected to routinely undergo surveillance mammography. In those treated with lumpectomy with or without RT, a cohort for whom annual surveillance mammography is recommended, $87 \%$ to $96 \%$ received mammography within 18 months, depending on geographic region. For women who underwent lumpectomy, those treated with RT were 2.5-fold more likely to undergo mammography than those who were not. Within the entire cohort, $32 \%$ underwent $\geq 1$ high-cost imaging examination. When stratified by treatment cohort and geographic region, $18 \%$ to $46 \%$ of all women in the study received a high-cost imaging examination within 18 months after diagnosis. Women who underwent a unilateral or bilateral mastectomy with RT were 6-fold more likely to be imaged with PET. Geographic variation existed for breast imaging and nonbreast imaging examinations.

Franc et al's research lends support to the growing body of literature regarding underuse and overuse of appropriate imaging examinations in the United States. For screening and surveillance, the goal is to detect primary, recurrent, or second primary breast cancers before symptoms develop, because early detection results in decreased mortality and treatment morbidity. Although screening mammography decreases breast cancer mortality by $20 \%$ to $40 \%$, only $58 \%$ of women aged 40 to 49 years and $71 \%$ of those aged 50 to 64 years received any type of mammogram in the preceding 2 years. ${ }^{3}$ To my knowledge, no data exist from randomized controlled trials regarding the benefit of different surveillance imaging strategies in breast cancer survivors. In observational studies of surveillance mammography, detection of recurrent or second primary breast cancers by mammography 
results in a $17 \%$ to $47 \%$ reduction in breast cancer mortality compared with detection by clinical examination. ${ }^{4}$ Yet, surveillance mammography remains underused. In a study of 1,762 survivors of early-stage breast cancer aged $\geq 65$ years, $82 \%$ underwent mammography in the first year after treatment but only $69 \%$ received mammography by the fourth year. ${ }^{5}$ Women diagnosed with stage II disease and those who underwent lumpectomy without RT were significantly less likely to undergo surveillance mammography, despite their increased risk of recurrence. ${ }^{5}$ Perhaps not surprisingly, women without follow-up visits to surgical or medical oncology were also less likely to receive surveillance imaging. ${ }^{5}$

In a separate study, among women aged $>65$ years without known metastatic disease, those diagnosed at a higher stage, at an older age, and with a longer time period since primary treatment were less likely to undergo surveillance mammography. ${ }^{6}$ In that study by Onega et al, ${ }^{6}$ only $89 \%$ and $81.5 \%$ of breast cancer survivors received mammography at 24 and 78 months, respectively. The frequency of surveillance mammography at an earlier time point is similar to the $87 \%$ to $96 \%$ reported by Franc et al, suggesting that adherence to the recommended surveillance imaging regimen is likely to further decline with increasing time from diagnosis and primary treatment.

Supplemental screening or surveillance breast MRI detects significantly more cancers than mammography alone (10-29 more cancers per 1,000 examinations in breast cancer survivors). ${ }^{4}$ Despite the increasing use of supplemental MRI for screening and surveillance, $<5 \%$ of women with an estimated lifetime risk of breast cancer of $\geq 20 \%$ and fewer than half of documented genetic mutation carriers received screening breast MRI as recommended by the American Cancer Society guidelines. ${ }^{7}$ Race and ethnicity, lower income or education level, rural residence, limited English proficiency, and lack of health insurance or higher out-of-pocket copayments contribute to the current screening and surveillance disparities. ${ }^{5}$ Because most states do not mandate insurance coverage for supplemental screening or surveillance modalities, existing disparities may worsen in the future.

Advanced imaging examinations, including CT, PET/CT, and MRI, have been overused in some groups of women at average or lower risk of developing primary, recurrent, or second primary breast cancer. Franc et al report on their data from 2010-2013. Changes in use may have occurred during and after the study period, because national efforts to curb inappropriate medical services, including imaging, came into the spotlight in 2012 when the American College of Physicians outlined 37 clinical situations in which medical testing did not add high value, including "In asymptomatic women with previously treated breast cancer, performing... imaging studies other than appropriate breast imaging."

The American Board of Internal Medicine Foundation and Consumer Reports spearheaded the "Choosing Wisely" initiative. ${ }^{9}$ In conjunction with ASCO, Choosing Wisely suggests that physicians "Don't perform surveillance...imaging (PET, CT, and radionuclide bone scans) for asymptomatic individuals who have been treated for breast cancer with curative intent." Published guidelines intended to increase appropriate use of medical services in patients with breast cancer and survivors are also available from NCCN (available at NCCN.org). Radiologists have been actively participating in these efforts from the beginning. Since 1993, the American College of Radiology (ACR) has convened expert panels to develop evidence-based appropriateness criteria (AC). ${ }^{10}$ As of April 25, 2018, 235 unique ACR AC topics have been published. Because of the rigorous methodology used for development of the AC, the ACR was approved as a qualified provider-led entity; thus, referring providers may consult the ACR AC before ordering advanced imaging services for Medicare patients, as mandated by the Protecting Access to Medicare Act of 2014. ${ }^{11}$ Implementation of clinical decision support mechanisms into electronic order entry systems has the potential to decrease inappropriate use, although data in patients with cancer are lacking.

In this issue of JNCCN, Franc et al remind us that appropriate use of medical services, including imaging, is critical to the delivery of high-value healthcare in patients with breast cancer. However, we all know that no magic bullet exists to solve this problem. We must galvanize support for research, administrative, and educational initiatives to address existing gaps. For example, we must identify and implement optimal imaging strategies for 
Niell

early detection of primary, second primary, and metastatic breast cancers, and then tailor those surveillance strategies based on risk of recurrence, life expectancy, and comorbid conditions; develop systems-based approaches to minimize underuse (eg, automated reminder systems within the electronic medical record for routinely recommended studies such as annual surveillance mammography); demand and support the integration of clinical decision support mechanisms that successfully curb overuse; eliminate barriers within and across vulnerable populations to ensure access to high-quality mammography and recommended supplemental screening modalities; engage survivorship clinics, primary care providers, and internists in addition to specialists; and support community outreach and educational programs to increase awareness. In brief, let's collaborate across disciplines, institutions, and countries to develop creative and innovative evidence-based solutions to optimize the appropriate use of imaging and other medical services in breast cancer survivors and in all patients.

\section{References}

1. Noone AM, Howlader N, Krapcho M, et al, editors. SEER Cancer Statistics Review, 1975-2015. Bethesda, MD: National Cancer Institute. Available at: https://seer.cancer.gov/csr/1975_2015/. Accessed June 20, 2018

2. National Cancer Policy Board. From Cancer Patient to Cancer Survivor: Lost in Transition. Washington, DC: National Academies Press; 2005.

3. National Center for Health Statistics. Health, United States, 2016: With Chartbook on Long-Term Trends in Health. Hyattsville, MD: US Department of Health and Human Services; May 2017. DHHS publication No. 2017-1232.

4. Lam DL, Houssami N, Lee JM. Imaging surveillance after primary breast cancer treatment. AJR Am J Roentgenol 2017;208:676-686.

5. Adesoye T, Schumacher JR, Neuman HB, et al. Use of breast imaging after treatment for locoregional breast cancer (AFT-01). Ann Surg Oncol 2018;25:1502-1511.

6. Onega T, Weiss J, Diflorio R, et al. Evaluating surveillance breast imaging and biopsy in older breast cancer survivors. Int J Breast Cancer 2012;2012:1-10.

7. Stout NK, Nekhlyudov L, Li L, et al. Rapid increase in breast magnetic resonance imaging use: trends from 2000 to 2011. JAMA Intern Med 2014;174:114-121.

8. Qaseem A, Alguire P, Dallas P, et al. Appropriate use of screening and diagnostic tests to foster high-value, costconscious care. Ann Intern Med 2012;156:147-149.

9. American Board of Internal Medicine Foundation. Choosing Wisely: promoting conversations between patients and clinicians. Available at: http://www.choosingwisely.org/. Accessed June 8, 2018.

10. ACR Appropriateness Criteria. American College of Radiology website. Available at: https://www.acr.org/ Clinical-Resources/ACR-Appropriateness-Criteria. Accessed June 15, 2018.

11. Protecting Access to Medicare Act of 2014, HR 4302, 113th Cong, S 218(b), 2014. Available at: https://www. congress.gov/113/plaws/pub193/PLAW-113pub193.pdf. 